\title{
DIAGNÓSTICOS DE ENFERMAGEM EM RECÉM-NASCIDO COM ALTERAÇÕES GLICÊMICAS
}

\author{
Samara Isabela Maia de Oliveira1, Nilba Lima de Souza², Rhuama Karenina Costa e Silva ${ }^{3}$
}

\begin{abstract}
RESUMO: Os diagnósticos de enfermagem resultam do agrupamento de dados coletados, que permitem ao enfermeiro o estabelecimento de intervenções na saúde do paciente. Esta pesquisa quantitativa e documental objetivou identificar os diagnósticos de enfermagem mais frequentes entre recém-nascidos com alterações glicêmicas internados em uma maternidade-escola em agosto de 2011. Os diagnósticos de enfermagem prioritários referem-se à Nutrição desequilibrada menos que as necessidades corporais, Risco de glicemia instável, Padrão ineficaz de alimentação do bebê, Risco de crescimento desproporcional, Paternidade ou maternidade prejudicada, Amamentação ineficaz, Troca de gases prejudicada, Amamentação interrompida e Risco de infecção. Foi observada predomínio de diagnósticos referentes ao Risco de crescimento desproporcional, Risco de infecção e Risco de glicemia instável. Ressalta-se a importância da utilização da Sistematização da Assitência de Enfermagem com o intuito de planejar o cuidado aos neonatos com distúrbios glicêmicos.
\end{abstract}

DESCRITORES: Neonato; Hipoglicemia; Hiperglicemia; Diagnóstico de enfermagem.

\section{NURSING DIAGNOSES IN NEW-BORNS WITH GLYCEMIC ALTERATIONS}

ABSTRACT: The nursing diagnoses result from the grouping of collected data, which allows the nurse to establish interventions in the patient's health. This quantitative and documental research aimed to identify the most frequent nursing diagnoses among new-borns with glycemic alterations, hospitalized in a maternity school in August 2011. The priority nursing diagnoses refer to unbalanced nutrition, of less than bodily needs, Risk of unstable glycemia, Inefficient pattern of feeding the baby, Risk of disproportionate growth, Impaired fatherhood and motherhood, Inefficient breastfeeding, Impaired gaseous exchange, Interrupted breastfeeding, and Risk of infection. A predominance was observed of diagnoses referent to Risk of disproportionate growth, Risk of infection, and Risk of unstable glycemia. The importance of using Systematization of Nursing Care is emphasized, with the aim of planning the care for neonates with glycemic disorders.

DESCRIPTORS: Neonate; Hypoglycemia; Hyperglycemia; Nursing diagnosis.

\section{DIAGNÓSTICOS DE ENFERMERÍA EN RECIÉN NACIDO CON ALTERACIONES GLICÉMICAS}

RESUMEN: Los diagnósticos de enfermería resultan del agrupamiento de datos recogidos, los cuales permiten al enfermero el establecimiento de intervenciones en la salud del paciente. Esta investigación cuantitativa y documental tuvo el objetivo de identificar los diagnósticos de enfermería más frecuentes entre recién nacidos con alteraciones glucémicas internados en una maternidad escuela en agosto de 2011. Los diagnósticos de enfermería prioritarios se asocian a la Nutrición desequilibrada menor que las necesidades corporales, Riesgo de glucemia inestable, Alimentación ineficaz del bebé, Riesgo de crecimiento desproporcional, Paternidad o maternidad perjudicada, Amamantamiento ineficaz, Cambio de gases perjudicada, Amamantamiento interrumpido y Riesgo de infección. Fue observado predominio de diagnósticos referentes al Riesgo de crecimiento desproporcional, Riesgo de infección y Riesgo de glucemia inestable. Se destaca la importancia de la utilización de la Sistematización de la Asistencia de enfermería con el intuito de planear el cuidado de los neonatos con disturbios de glucemia.

DESCRIPTORES: Neonato; Hipoglucemia; Hiperglucemia; Diagnóstico de enfermería.

${ }^{1}$ Enfermeira. Especialista em Enfermagem do Trabalho. Membro do Grupo de Pesquisa: Cuidado de Enfermagem em Diferentes Fases da Vida da Universidade Federal do Rio Grande do Norte.

${ }^{2}$ Enfermeira. Doutora em Enfermagem. Professora do Departamento de Enfermagem e do Programa de Pós-Graduação em Enfermagem na Vigilância à Saúde da UFRN. Membro do Grupo de Pesquisa: Cuidado de Enfermagem em Diferentes Fases da Vida.

${ }^{3}$ Enfermeira. Mestranda pelo Programa de Pós-Graduação em Enfermagem da UFRN. Membro do Grupo de Pesquisa: Cuidado de Enfermagem em Diferentes Fases da Vida.

Autor correspondente:

Samara Isabela Maia de Oliveira

Universidade Federal do Rio Grande do Norte

Rua Maria Quitéria, 3586 - 59064-680 - Natal-RN-Brasil

E-mail: aramas.maia@gmail.com
Recebido: 13/12/2012

Aprovado: 07/10/2013

Cogitare Enferm. 2013 Out/Dez; 18(4):702-8 


\section{INTRODUÇÃO}

A Sistematização da Assistência em Enfermagem (SAE) surgiu como um meio norteador das ações a serem executadas para o paciente, de forma dinâmica e objetiva; organiza o trabalho quanto ao método, pessoal e instrumentos, tornando possível a implementação do Processo de Enfermagem (PE). Estes instrumentos estão regulamentados pela Resolução do Conselho Federal de Enfermagem n. 385/2009 que dispõe sobre seu emprego em todos os ambientes da prática profissional ${ }^{(1)}$.

O PE é um instrumento metodológico de prestação de cuidado constituído por um conjunto de etapas que permitem identificar situações de saúde/doença do indivíduo. As etapas realizadas sequencialmente embasam a seleção e a implementação de ações e o acompanhamento de seus resultados. É instituído pelas seguintes fases: histórico (investigação), diagnóstico de enfermagem (DE), planejamento, implementação e avaliação ${ }^{(1-2)}$.

Os DE se baseiam no agrupamento de dados coletados para instituição de uma informação diagnóstica sobre o paciente para o cuidado; contribuem para base do conhecimento da profissão e o estabelecimento de cuidados específicos a uma determinada situação ou indivíduo. O uso de uma taxonomia, sistema de classificação da linguagem profissional para DE contribui para futuros estudos sobre o tema. A mais comumente utilizada é a Taxonomia II da North American Nursing Diagnosis Association - NANDA ${ }^{(2-4)}$.

Os DE permitem que o enfermeiro estabeleça intervenções priorizando as situações de agravamento, risco ou predisponentes ao comprometimento da saúde do paciente. Uma dessas situações de risco é encontrada em recém-nascidos (RN) acometidos por distúrbios glicêmicos, por carecerem de uma atenção especial voltada aos sinais, sintomas e prevenção dos agravos decorrentes das alterações com intervenções realizadas pela equipe de enfermagem.

Neste contexto, a SAE direciona a elaboração de plano de cuidados, contribui com a melhoria da assistência de enfermagem e redução da morbidade e mortalidade, inclusive em pacientes com distúrbios glicêmicos. Esses são, muitas vezes, a causa de agravos metabólicos em RN; configuram fatores de risco de mortalidade infantil e estão relacionados com agravamento de lesões cerebrais. $\mathrm{O}$ aporte insatisfatório de nutrientes e de dispensação das reservas de glicose existentes ocasionam a hipoglicemia, comprometendo o desenvolvimento adequado dos neonatos ${ }^{(5)}$.

A hiperglicemia ocorre em $20 \%$ a $85 \%$ dos $\mathrm{RN}$ e não há um consenso sobre a sua exata definição. Sugere-se como ponto de corte para hiperglicemia níveis de glicose de $145 \mathrm{mg} / \mathrm{dl}$. A glicemia sérica elevada está entre os mais importantes distúrbios na população pediátrica, e é altamente prevalente em crianças internadas em terapia intensiva. As taxas de incidência são variáveis e altas, tanto para picos isolados quanto para hiperglicemia persistente durante a internação ${ }^{(6-7)}$.

A hipoglicemia neonatal acomete de $3 \%$ a $43 \%$ dos neonatos e ocorre principalmente nas primeiras horas de vida. A glicose é ofertada ao feto por gradiente de concentração por meio da placenta. Dessa forma, a glicemia do feto está diretamente relacionada à glicemia materna, ficando em torno de 50 a $55 \mathrm{mg} / \mathrm{dL}$. Pode-se ponderar que esses valores norteiam os níveis de normalidade no $\mathrm{RN}$ pré-termo e que os valores abaixo se caracterizam por hipoglicemia ${ }^{(6,8)}$. A adaptação metabólica no nascimento envolve a mobilização de depósitos de glicogênio, síntese hepática de glicose de outros substratos e a produção de combustíveis cerebrais alternativos, tais como os corpos cetônicos. Os episódios de hipoglicemia podem ser devido ao esgotamento dos estoques de glicogênio perinatal, enquanto que os episódios hiperglicêmicos podem estar ligados à liberação de hormônios relacionados ao estresse $\mathrm{e}^{(9)}$.

Um grande desafio tem sido determinar os danos cerebrais que podem se desenvolver quando as concentrações de glicose plasmática caem para valores mais baixos e permanecem baixos por períodos prolongados. Não se sabe qual valor, quantas vezes, ou por quanto tempo as concentrações de glicose plasmática baixas devem ocorrer antes que haja danos neuronais irreversíveis $^{(10)}$. Neste sentido, os DE revelam condições de saúde que exigem atuação imediata da equipe para que não sejam gerados danos ao desenvolvimento dos RN. A literatura aponta que a hipoglicemia não tratada ocasiona hipotonia, hipotermia, convulsões, cianose, e até insuficiência cardíaca. A hiperglicemia pode levar o neonato a um desequilíbrio osmótico, levando à alteração do volume cerebral devido ao deslocamento de líquido, podendo ocasionar hemorragia intraventricular, além de ser um grande precursor de diabetes em adolescentes ${ }^{(9-10)}$.

O distúrbio glicêmico em neonatos é um tema bastante discutido na literatura em virtude dos agravos que essas alterações podem acarretar para o desenvolvimento dos RN. Esses distúrbios requerem atenção especial da enfermagem por predisporem a danos 
neurológicos, dependendo da sua gravidade. A partir de estudos, e conhecendo as alterações glicêmicas neonatais, podem-se elaborar DE que norteiem o trabalho da equipe de enfermagem que presta assistência a esses RN, visando reduzir as complicações preveníveis advindas desses agravos. Baseado nisso, este trabalho objetivou identificar os Diagnósticos de Enfermagem mais frequentes entre recém-nascidos com alterações glicêmicas internados em uma maternidade-escola.

\section{MÉTODO}

Estudo do tipo quantitativo, descritivo, documental, realizado na Maternidade Escola Januário Cicco, unidade pertencente à Universidade Federal do Rio Grande do Norte que é referência para gravidez e nascimento de risco no estado. A população foi composta por todos os prontuários dos $\mathrm{RN}$ com história de alterações glicêmicas, nascidas de parto cesáreo, hospitalizados no campo de pesquisa, no período de agosto de 2011. Atendendo aos critérios de inclusão, foram analisados os prontuários de RN que apresentaram alterações glicêmicas, com dieta zero, oral, por gavagem, copo e/ou sucção; foram excluídos os prontuários dos neonatos com complicações glicêmicas associadas à atresia de esôfago e à má-formação congênita, visto que comprometeriam os dados da avaliação. A pesquisa obteve aprovação no comitê de ética sob protocolo número 602/11 e autorização do diretor do campo de estudo.

No primeiro momento, fez-se uma análise dos prontuários, por meio de leitura minuciosa, investigando todos os registros de $\mathrm{RN}$ de parto cesáreo do mês de agosto de 2011. Realizou-se a distinção dos registros maternos e neonatais com alterações glicêmicas, onde 30 prontuários foram selecionados, identificando dados de caracterização dos RN, informações obstétrico-neonatais, agravos maternos, valores de glicemia capilar, tratamento, os sinais e sintomas e as ações de cuidado registradas pelos diferentes profissionais da equipe, no intuito de elencar as pistas diagnósticas. Os dados foram descritos quantitativamente e apresentados no formato de tabelas, registrados em um banco de dados e analisados através do Statistical Package for the Social Sciences - SPSS 17.0 e analisadas com auxílio de estatística descritiva.

No segundo momento, houve a correlação dos indicativos diagnósticos com os dados encontrados, utilizando raciocínio clínico e definições elucidadas na Taxonomia-II NANDA, para a elaboração dos Diagnósticos de Enfermagem ${ }^{(4)}$. Foram atribuídos três diagnósticos prioritários relacionados aos distúrbios glicêmicos e posteriormente agrupados em percentuais. A elaboração diagnóstica foi realizada pelos enfermeiros participantes do estudo, com supervisão da orientadora da pesquisa e colaboração dos enfermeiros da Unidade de Terapia Intensiva Neonatal.

\section{RESULTADOS}

De um total de $30 \mathrm{RN}$ foi observada predominância do sexo feminino com $63,3 \%$; a maior parte do grupo estudado teve idade gestacional entre 33 a 36 semanas, caracterizando a amostra com maioria de neonatos pré-termos.

Dos RN com distúrbios da glicose, $50 \%$ pesaram entre 1501 gramas a 2500 gramas ao nascer; $23,3 \%$ pesaram menos que 1500 gramas e $26,7 \%$ pesaram mais que 2500 gramas, como visto na tabela 1 .

A maior parcela de $\mathrm{RN}$ foi acometida por hipoglicemia $(66,7 \%)$, sendo a associação da hipoglicemia e hiperglicemia representada por $23,3 \%$ e, por último, a hiperglicemia com 10\%. Em $60 \%$ dos eventos de distúrbios glicêmicos, o local de internação destinado foi

Tabela 1 - Características dos recém-nascidos com alterações glicêmicas. Natal, 2011

\begin{tabular}{lcc}
\hline Característica & $\mathbf{N}$ & $\mathbf{\%}$ \\
\hline Sexo & 11 & 36,7 \\
Masculino & 19 & 63,3 \\
Feminino & & \\
Idade Gestacional & 05 & 16,7 \\
De 28 a 32 semanas & 13 & 43,3 \\
De 33 a 36 semanas & 12 & 40,0 \\
> 37 semanas & & \\
Peso ao Nascer & 07 & 23,3 \\
Até 1.500 gramas & 15 & 50,0 \\
De 1501 g a 2500 gramas & 08 & 26,7 \\
\hline 2500 gramas &
\end{tabular}

o alojamento conjunto, e $40 \%$ deles foram admitidos na UTI Neonatal. A máxima frequência do período de internação hospitalar esteve entre um e sete dias, com $56,7 \%$, como visto na tabela 2 .

Entre os agravos materno-gestacionais mais frequentes foram observados a Doença Hipertensiva Específica da Gestação (DHEG) em 40\%, amniorrexe prematura em $26,7 \%$ dos casos, Infecção do Trato Urinário eram em $20 \%$ das gestantes e apenas $10 \%$ de mães portadoras de Diabetes Melitus.

Os DE mais prevalentes em relação aos distúrbios 
glicêmicos estavam relacionados com fatores nutricionais, dificuldades na amamentação, ao peso inadequado comparado ao percentil para a idade, estados de gravidade e disfunções respiratórias, bem como aos riscos de infecção aos quais estavam expostos. Destarte, os domínios mais frequentes foram: Domínio 2- Nutrição em 33,33\%, Domínio 7- Relacionamento de Papel com 33,33\%, Domínio 3- Eliminação e Troca presente em 11,11\%, Domínio 11-Segurança/Proteção

Tabela 2 - Características da internação dos recém-nascidos com alterações glicêmicas. Natal, 2011

\begin{tabular}{lcc}
\hline Característica & N & \% \\
\hline Distúrbio Glicêmico & 20 & 66,7 \\
Hipoglicemia & 03 & 10 \\
Hiperglicemia & 07 & 23,3 \\
Hiperglicemia e & & \\
Hipoglicemia & & \\
Local de Internação & 12 & 40,0 \\
UTI Neonatal & 18 & 60,0 \\
Alojamento Conjunto & & \\
Tempo de Internação & 17 & 56,7 \\
Até 7 dias & 07 & 23,3 \\
De 8 a 15 dias & 02 & 6,7 \\
De 16 a 30 dias & 04 & 13,3 \\
>31 dias & &
\end{tabular}

\section{DISCUSSÃO}

Os dados mostram que o tempo de internação e a gravidade do distúrbio variaram de acordo com a idade gestacional e peso do RN. Os neonatos prematuros e de baixo peso são predisponentes a alterações glicêmicas, visto que estes não possuem reservas energéticas adequadas à situação do nascimento. Os RN macrossômicos recebem, muitas vezes, glicose materna em grande quantidade, justificando o sobrepeso, bem como os episódios hipoglicêmicos por hiperinsulinemia. Desse modo, percebe-se a prevalência do diagnóstico Risco de crescimento desproporcional definido por risco de crescimento acima do $97^{\circ}$ percentil ou abaixo do $3^{\circ}$ percentil para a idade, cruzando duas faixas percentis em $56,66 \%$ dos neonatos. A enfermagem deve identificar precocemente os $\mathrm{RN}$ com evidências para risco de crescimento desproporcional, e ser vigilante frente à variação glicêmica detectável por meio da avaliação clínica e da glicemia capilar.

O Risco de glicemia instável esteve presente em $43,33 \%$ dos neonatos; é o DE definido por risco de variação dos níveis de glicose no sangue em relação evidenciado em 11,11\% e o Domínio 13- Crescimento e Desenvolvimento identificado em $11,11 \%$ dos RN.

Os DE encontrados foram: Nutrição desequilibrada menos que as necessidades corporais; Risco de glicemia instável; Padrão ineficaz de alimentação do bebê; Risco de crescimento desproporcional; Paternidade ou maternidade prejudicada; Amamentação ineficaz; Troca de gases prejudicados; Amamentação interrompida; e Risco de infecção (Tabela 3).

Tabela 3 - Diagnósticos de enfermagem dos recém-nascidos com alterações glicêmicas. Natal, 2011

\begin{tabular}{lr}
\hline Diagnóstico de Enfermagem & \% \\
\hline Risco de crescimento desproporcional & 56,66 \\
Risco de infecção & 53,33 \\
Risco de glicemia instável & 43,33 \\
Nutrição desequilibrada menos que as & 40 \\
necessidades corporais & 36,66 \\
Troca de gazes prejudicada & 30 \\
Paternidade ou maternidade & 30 \\
prejudicada & 26,66 \\
Amamentação interrompida & 23,33 \\
Amamentação ineficaz & \\
Padrão ineficaz de alimentação do bebê &
\end{tabular}

aos parâmetros normais, referente a fatores de risco como a perda de peso, estado de saúde física, estresse, ingestão alimentar, monitorização inadequada da glicemia. Sua alta incidência justifica-se pelas alterações glicêmicas sofrerem influência do agravamento do quadro clínico, ocorrência de procedimentos invasivos e prognóstico em que o neonato se encontra. Os episódios hiperglicêmicos estiveram, em grande parte da amostra, relacionados à condição comprometida de saúde. A monitorização, bem como o controle adequado da glicemia, é um trabalho da equipe de saúde e, neste contexto, compete à enfermagem a aferição da glicose capilar ao serem percebidos sinais de alterações clínicas no RN, a fim de evitar as consequências de alteração glicêmica não observada e tratada.

Alguns neonatos apresentaram desconforto respiratório precoce, os quais eram submetidos a jejuns prolongados, propiciando a ocorrência da hipoglicemia. Nesse contexto, foram atribuídos os diagnósticos: Troca de gases prejudicada em $36,66 \%$ dos bebês, definido pelo excesso ou déficit na oxigenação e ou na eliminação de dióxido de carbono na membrana alvéolo capilar, relacionado a desequilíbrio na ventilação/ 
perfusão e mudança na membrana alvéolo capilar. E Amamentação interrompida como a quebra da continuidade do processo de amamentação em resultado da incapacidade ou inconveniência de colocar a criança no peito para mamar, relacionado à doença da criança. Este último ocorreu em $30 \%$ dos RN; a enfermagem deve dar atenção à possibilidade de hipoglicemia neonatal quando constatar quaisquer intercorrências no aleitamento materno.

Risco de infecção é o diagnóstico definido por risco aumentado de ser invadido por organismos patológicos, relacionado à desnutrição, exposição ambiental aumentada a patógenos, procedimentos invasivos, ruptura prematura das membranas amnióticas, encontrado em $53,33 \%$ dos RN. Os dados mostraram grande quantidade de parturientes com bolsa rota acima de seis horas, favorecendo infecções. A amniorrexe prematura esteve presente em $26,7 \%$ dos casos. Além disso, verificou-se que muitos neonatos foram submetidos a grande quantidade de procedimentos invasivos.

Partes dos diagnósticos encontrados remetem à nutrição desequilibrada em $\mathrm{RN}$, contribuindo para os distúrbios glicêmicos. A Nutrição desequilibrada menos que as necessidades corporais foi o diagnóstico encontrado em $40 \%$ dos bebês, sendo definido por ingestão insuficiente de nutrientes para satisfazer as necessidades metabólicas, e relacionado à capacidade prejudicada de ingerir alimentos, a fatores biológicos e psicológicos. É imprescindível a observação atenta da equipe de enfermagem em relação aos sinais hipoglicêmicos manifestados pelo RN, além de observar os padrões de amamentação, apojadura do leite materno, e padrões de sucção do neonato, quando esse é colocado para amamentar ${ }^{(8)}$.

Dificuldades na amamentação estão relacionadas como um fator relevante para a alteração glicêmica. Desse modo, o diagnóstico Amamentação ineficaz, definido por insatisfação ou dificuldade que a mãe, o bebê ou a criança experimenta com o processo de amamentação e está relacionado à anomalia de peito materno, déficit no conhecimento, interrupção na amamentação prematuridade, reflexo de sucção da criança insatisfatória; esteve presente em $26,66 \%$ dos neonatos. A equipe de enfermagem deve dar atenção às situações variantes da amamentação, às dúvidas, às pegas incorretas, às lesões mamilares, e dificuldade de ordenha. E deve desenvolver atividades junto às puérperas, visando orientá-las e capacitá-las na tarefa de amamentar, observando a interação da mãe com o filho, contribuindo para uma amamentação eficaz ${ }^{(8)}$.
Foram observados diversos registros referentes à sucção débil, à ausência de colostro, anatomia desfavorável do mamilo e ao desconhecimento materno quanto à frequência das mamadas. Nesse contexto, foi observado o diagnóstico Padrão ineficaz de alimentação do bebê, definido como a capacidade prejudicada de um bebê de sugar ou de coordenar a resposta sucção deglutição, resultando em nutrição oral inadequada para as necessidades metabólicas; Relacionado à prematuridade, anomalia anatômica, estado de jejum oral prolongado e prejuízo neurológico, foi constatado em $23,33 \%$ da amostra. A equipe de saúde deve estar capacitada para orientar e incentivar o aleitamento materno, observar e avaliar a mamada ao peito, avaliar a mama puerperal e orientar quanto à prevenção das patologias.

Neste estudo, poucos casos de alterações da glicose foram relacionados ao Diabetes Gestacional, apenas $10 \%$ deles. Isso comprova a relação que existe entre o controle glicêmico e a morbimortalidade maternofetal. O controle glicêmico durante os períodos pré e concepcional, principalmente antes da $32^{\mathrm{a}}$ semana de gestação, é de especial importância para a redução das complicações perinatais ${ }^{(11-12)}$.

Pouco é explorada na literatura a relação entre as síndromes hipertensivas da gravidez e as alterações glicêmicas nos recém-nascidos. Alguns autores falam sobre a possibilidade de ocorrência da hipoglicemia neonatal associada ao uso de betabloqueadores maternos. Sobretudo, sabe-se que a DHEG é fator de risco para disfunção placentária e restrição do crescimento intrauterino; e esteve presente em $40 \%{ }^{(13)}$.

Outros agravos materno-gestacionais foram frequentes, dos quais obteve destaque a infecção do trato urinário em $20 \%$ das gestantes. É importante salientar que existe um quantitativo de puérperas que, acometidas por tais agravos gestacionais, necessita de cuidados intensivos e utilização de drogas, o que impossibilitou o acompanhamento do neonato.

Neste contexto, não se pode esquecer a presença materna na reabilitação dos RN para estabilização da alteração glicêmica, bem como sua necessidade de conhecimento sobre a patologia para o favorecimento do cuidado. Desse modo, o diagnóstico Paternidade ou maternidade prejudicada, definido como a incapacidade de cuidados primários de criar, manter ou reaver um ambiente que promova o ótimo crescimento e desenvolvimento da criança, relacionado à doença, nascimento prematuro, conhecimento deficiente sobre a manutenção da saúde da criança, esteve evidenciado em $30 \%$ dos RN. 


\section{CONCLUSÕES}

O desenvolvimento desta pesquisa favoreceu a identificação das necessidades de cuidados aos RN com distúrbios glicêmicos e a atribuição diagnóstica. Foi observada a predominância de diagnósticos referentes ao Risco de crescimento desproporcional, Risco de infecção e Risco de glicemia instável, e está pertinente à prevalência de $\mathrm{RN}$ prematuros, com baixo peso, em condições de agravamento clínico, submetidos a procedimentos invasivos com exposição a patógenos, implicando uma atenção especial do enfermeiro a todos os eventos.

Os diagnósticos Amamentação interrompida, Amamentação ineficaz, Padrão ineficaz de alimentação do bebê e Nutrição desequilibrada menos que as necessidades corporais se fizeram presentes em muitos dos casos e indicam a necessidade de atuação da enfermagem frente a orientações nutricionais e vigilância no ato de amamentar.

Os dados obtidos remetem à utilização da SAE nas situações de alterações glicêmicas, a ser utilizada pelo enfermeiro com o intuito de planejar, cuidadosamente, a assistência de enfermagem. $\mathrm{O}$ estudo mostrou que muitos fatores podem estar associados para a disposição de distúrbio glicêmico em neonatos. Desse modo, os DE devem atender às necessidades de cuidado $\mathrm{e}$ intervenções voltadas ao estabelecimento e manutenção dos padrões normais, bem como controlar as complicações resultantes dos padrões alterados.

\section{REFERÊNCIAS}

1. Conselho Federal de Enfermagem. Resolução n. 385, de 15 de outubro de 2009. Dispõe sobre a Sistematização da Assistência de Enfermagemea implementação do Processo de Enfermagem em ambientes, públicos ou privados, em que ocorre o cuidado profissional de Enfermagem, e dá outras providências. Brasília: COFEN; 2009.

2. Santana LL, Mazza VA, Taube SAM, Sarquis LMM. Diagnósticos e intervenções de enfermagem em unidade de pronto atendimento à luz das necessidades humanas básicas. Cogitare enferm. [Internet] 2011;16(4) [acesso em 13 set 2012]. Disponível: http://ojs.c3sl.ufpr. br/ojs2/index.php/cogitare/article/view/25436/17057

3. Santos WLS, Nakatani AYK, SantanaRF, Bachion MM. Diagnósticos de enfermagem identificados na alta hospitalar de Idosos. Cogitare enferm. [Internet] 2009;14(2) [acesso em 12 ago 2012]. Disponível: http://ojs.c3sl.ufpr. br/ojs2/index.php/cogitare/article/view/15622/10393
4. North American Nursing Diagnosis Association-NANDA. Diagnósticos de enfermagem da NANDA: definições e classificação 2005-2006. Porto Alegre: Artmed; 2006.

5. Liberatore Junior RR, Martinelli Junior CE. Hipoglicemia hiperinsulinêmica da infância. Arq. bras. endocrinol. metab. [Internet] 2011;44(3) [acesso em 05 mai 2012]. Disponível: http://dx.doi.org/10.1590/ S0004-27302011000300001

6. Brasil. Atenção à saúde do recém-nascido: guia para os profissionais de saúde. v. 4. Cuidados com o recémnascido pré-termo. Brasília: Ministério da Saúde; 2011.

7. Mekitarian Filho E, Carvalho WB, Troster EJ. Hiperglicemia e morbimortalidade em crianças graves: análise crítica baseada em revisão sistemática. Rev. Assoc. Méd. Bras. [Internet] 2009;55(4) [acesso em 11 set 2012]. Disponível: http://dx.doi.org/10.1590/S010442302009000400026

8. Freitas P. Prevalência e fatores associados à hipoglicemia transitória em recém-nascidos internados em um Hospital Amigo da Criança [dissertação]. São Paulo (SP): Escola de Enfermagem da Universidade de São Paulo; 2009.

9. Nadeem M, Deirdre MM, Geraldine BB, Eugene MD, Cornelius RAA. Early blood glucose profile and neurodevelopmental outcome at two years in neonatal hypoxic-ischaemic encephalopathy. BMC pediatr. [Internet] 2011;11(10) [acesso em 06 mai 2012]. Disponível: http://www.ncbi.nlm.nih.gov/pmc/articles/PMC3040139/

10. Hay Junior WW, Rozance PJ. Continuous Glucose Monitoring for Diagnosis and Treatment of Neonatal Hypoglycemia. JPediatr. [Internet] 2010;156(2) [acessoem 10 mai 2012]. Disponível: http://www.jpeds.com/article/ S0022-3476\%2810\%2900307-0/fulltext \#article-outline

11. Chaves EGS, Franciscon PM, Nascentes GAN, Paschoini MC, Silva AP, Borges MF. Estudo retrospectivo das implicações maternas, fetais e perinatais em mulheres portadoras de diabetes, em 20 anos de acompanhamento no Hospital Escola da Universidade Federal do Triângulo Mineiro. Arq Bras Endocrinol Metab. [Internet] 2010;54(7):620 [acesso em 10 set 2012]. Disponível: http://dx.doi.org/10.1590/S0004-27302010000700006

12. Silva JC, Bertini AM, Ribeiro TE, Carvalho LS, Melo MM, Barreto NL. Fatores relacionados à presença de recém-nascidos grandes para a idade gestacional em gestantes com diabetes mellitus gestacional. Rev. Bras. Ginecol. Obstet. [Internet] 2009;31(1) [acesso em 12 mai 2012]. Disponível: http://dx.doi.org/10.1590/S010072032009000100002 
13. Freitas P, Matos CV, Kimura AF. Perfil das mães de neonatos com controle glicêmico nas primeiras horas de vida. Rev Esc Enferm USP. [Internet] 2010;44(3). [acesso em 17 ago 2012]. Disponível: http://dx.doi. org/10.1590/S0080-62342010000300012 